\title{
Long Noncoding RNA Expression Signatures of Metastatic Nasopharyngeal Carcinoma and Their Prognostic Value
}

\author{
Wei Zhang, ${ }^{1,2}$ Lin Wang, ${ }^{3,4}$ Fang Zheng, ${ }^{5}$ Ruhai Zou, ${ }^{6}$ Changqing Xie, ${ }^{7}$ Qiannan Guo, \\ Qian Hu, ${ }^{8}$ Jianing Chen, ${ }^{1,2}$ Xing Yang, ${ }^{8}$ Herui Yao, ${ }^{8}$ Erwei Song, ${ }^{1,2}$ and Yanqun Xiang ${ }^{3,4}$ \\ ${ }^{1}$ Breast Tumor Center, Sun Yat-Sen Memorial Hospital, Sun Yat-Sen University, 107 Yanjiang West Road, Guangzhou, \\ Guangdong 510120, China \\ ${ }^{2}$ Key Laboratory of Malignant Tumor Gene Regulation and Target Therapy of Guangdong Higher Education Institutes, \\ Sun Yat-Sen Memorial Hospital, Sun Yat-Sen University, 107 Yanjiang West Road, Guangzhou, Guangdong 510120, China \\ ${ }^{3}$ Department of Nasopharyngeal Carcinoma, Sun Yat-Sen University Cancer Center, 651 Dongfeng East Road, Guangzhou, \\ Guangdong 510060, China \\ ${ }^{4}$ State Key Laboratory of Oncology in Southern China, Guangzhou, China \\ ${ }^{5}$ Medical Research Center, Sun Yat-Sen Memorial Hospital, Sun Yat-Sen University, 107 Yanjiang West Road, Guangzhou, \\ Guangdong 510120, China \\ ${ }^{6}$ Department of Ultrasonography, Sun Yat-Sen University Cancer Center, 651 Dongfeng East Road, Guangzhou, \\ Guangdong 510060, China \\ ${ }^{7}$ Department of Internal Medicine, Brody School of Medicine, Vidant Medical Center, East Carolina University, \\ Greenville, NC, USA \\ ${ }^{8}$ Department of Oncology, Sun Yat-Sen Memorial Hospital of Sun Yat-Sen University, 107 Yanjiang West Road, Guangzhou, \\ Guangdong 510120, China
}

Correspondence should be addressed to Herui Yao; yaoherui@163.com, Erwei Song; songew@mail.sysu.edu.cn, and Yanqun Xiang; yanqun.xiang@gmail.com

Received 24 June 2014; Accepted 2 September 2014

Academic Editor: Murat Gokden

Copyright (C) 2015 Wei Zhang et al. This is an open access article distributed under the Creative Commons Attribution License, which permits unrestricted use, distribution, and reproduction in any medium, provided the original work is properly cited.

Long noncoding RNAs (lncRNAs) have recently been found to play important roles in various cancer types. The elucidation of genome-wide lncRNA expression patterns in metastatic nasopharyngeal carcinoma (NPC) could reveal novel mechanisms underlying NPC carcinogenesis and progression. In this study, lncRNA expression profiling was performed on metastatic and primary NPC tumors, and the differentially expressed lncRNAs between these samples were identified. A total of 33,045 lncRNA probes were generated for our microarray based on authoritative data sources, including RefSeq, UCSC Knowngenes, Ensembl, and related literature. Using these probes, 8,088 lncRNAs were found to be significantly differentially expressed ( $\geq 2$-fold). To identify the prognostic value of these differentially expressed lncRNAs, four lncRNAs (LOC84740, ENST00000498296, AL359062, and ENST00000438550) were selected; their expression levels were measured in an independent panel of 106 primary NPC samples via QPCR. Among these lncRNAs, ENST00000438550 expression was demonstrated to be significantly correlated with NPC disease progression. A survival analysis showed that a high expression level of ENST00000438550 was an independent indicator of disease progression in NPC patients $(P=0.01)$. In summary, this study may provide novel diagnostic and prognostic biomarkers for NPC, as well as a novel understanding of the mechanism underlying NPC metastasis and potential targets for future treatment.

\section{Introduction}

Nasopharyngeal carcinoma (NPC), a squamous cell carcinoma that occurs in the epithelial lining of the nasopharynx, displays a characteristic geographic and racial distribution worldwide. NPC is a rare malignant tumor in Western countries with an incidence of less than 1/100,000; however, the incidence of NPC was reported to be greater than 
20/100,000 in southern China, especially among the Cantonese population living in the central region of Guangdong Province $[1,2]$. The histological profile of NPC varies between endemic and nonendemic areas. For example, the tumors from more than $95 \%$ of NPC patients in high-incidence areas of China are undifferentiated nonkeratinizing carcinoma, whereas those from patients of Western descent, such as Caucasian, African-American, and Hispanic patients, are predominantly keratinizing squamous cell carcinoma [3-5]. According to the WHO histological profile, NPC among Chinese patients accounts for the majority of nonkeratinizing carcinomas, including $55.9 \%$ of the differentiated nonkeratinizing carcinomas and $58.0 \%$ of the undifferentiated nonkeratinizing carcinomas. This difference is attributed to the multifactorial etiology of NPC, which includes genetic factors, viral infection, the environment, and dietary habits [5-12]. The cure rate of NPC has improved significantly since the development of radiation technology and chemotherapy. However, distant metastasis remains the primary reason for treatment failure $[3,11,13]$. It is necessary to identify the specific molecular mechanisms that contribute to the pathogenesis and progression of NPC metastasis.

Recent studies suggest that noncoding RNAs (ncRNAs) constitute a large proportion of genome-encoded transcripts [14-16]. There is increasing evidence confirming that ncRNA performs biological functions in both cis- and trans-gene regulation, especially among higher eukaryotes [16-19]. Due to their functional relevance, ncRNAs have been categorized into housekeeping and regulatory ncRNAs [15]. Long noncoding RNAs (lncRNAs with a length of more than 200 nucleotides) comprise a majority of regulatory ncRNAs $[15,16,20]$. Many lncRNAs are highly conserved and are involved in diverse cellular functions, such as epigenetic regulation [21-23]. IncRNAs have been demonstrated to play crucial roles in dosage compensation, genome imprinting, $\mathrm{X}$ chromosome inactivation, chromatin modification, and whole-genome rearrangement $[17,18,21,24,25]$. The dysregulated expression of $\operatorname{lncRNAs}$ has been identified in a variety of diseases, including different types of cancer [26]; this observation suggests that aberrant lncRNA expression may represent a major contributor to carcinogenesis and cancer progression [17, 27]. For example, HOTAIR and ANRIL act as cancer regulators in carcinogenesis and cancer progression $[17,28]$. HOTAIR expression levels increase with clinical stage progression in NPC; NPC patients with high HOTAIR levels have a poor prognosis for overall survival [29]; metastasis-associated lung adenocarcinoma transcript (MALAT-1), PANDA, and ncRNA-DHFR regulate DNA damage, the cell cycle, alternative splicing, and tumor progression [30, 31]. Based on microarray analysis, the H19 gene is strongly expressed in undifferentiated NPC. Furthermore, H19 is highly expressed in an undifferentiated human NPC cell line. H19 plays a role in the differentiation of human NPC cells and the transcriptional silencing of imprinted genes [32]. LINC00312, also named NAG7 (NPC-associated gene 7), is a lincRNA expressed in the cytoplasm of nasopharyngeal epithelial cells. LINC00312 is expressed in 51.4\% of NPC samples and $78.4 \%$ of noncancerous nasopharyngeal epithelia samples $(P<0.001)$ [33]. Compared with noncancerous nasopharyngeal epithelial tissues, LINC00312 is significantly downregulated in NPC tissues. LINC00312 could be used as a biomarker for NPC metastasis, progression, and prognosis. Based on rematching and reannotation of the existing microarray datasets, five lncRNAs were selected to validate the differential expression of lncRNAs in both primary and recurrent nasopharyngeal carcinoma compared with noncancerous nasopharyngeal epithelia [34]. However, most of the differentially expressed lncRNAs have not been functionally characterized. We suspect that some of these lncRNAs play roles in NPC progression and that some are candidate biomarkers for the diagnosis or prognosis of NPC. The novel molecular mechanisms by which lncRNAs regulate carcinogenesis and metastasis are expected to be elucidated.

In the present study, we performed lncRNA expression profiling on metastatic and primary NPC tumors and identified differentially expressed lncRNAs that could show altered expression prior to or during the invasion-metastasis process. Further investigation validated that the expression level of the lncRNA ENST00000438550 was an independent prognostic marker in NPC patients.

\section{Materials and Methods}

2.1. Patients and Tissue Specimens. From July 2010 to November 2012, a total of 110 primary NPC samples and 3 metastatic NPC samples with confirmed pathology were collected from Sun Yat-Sen University Cancer Center. All of the samples were excess discarded tissues from diagnostic procedures. Three NPC metastatic tissue samples were collected via needle biopsy of bone metastatic sites of NPC patients. Among the 110 primary NPC samples, 4 of them were randomly selected for lncRNA microarray analysis. The remaining 106 primary NPC samples underwent QPCR. The tumor tissues from each subject were snap-frozen in liquid nitrogen immediately after biopsy. Written informed consent was obtained from all patients. The research ethics committee of Sun Yat-Sen University Cancer Center approved this study. No patients had received therapy prior to biopsy. The TNM classification of the patients was determined according to the criteria of the American Joint Committee on Cancer (AJCC 7 th edition). The detailed clinical information corresponding to the seven NPC patient samples used for microarray analysis is presented in Table S01 in the Supplementary Material available online at http://dx.doi.org/10.1155/2015/618924.

2.2. RNA Extraction. Total RNA was extracted from 113 snapfrozen samples using TRIzol reagent (Invitrogen) according to the manufacturer's protocol. The sample quality was evaluated using a Nano Drop ND-1000 spectrophotometer and standard denaturing agarose gel electrophoresis.

2.3. Microarray and Computational Analyses. For microarray analysis, the previously prepared total RNA from each sample was purified after rRNA removal (mRNA-ONLY Eukaryotic mRNA Isolation Kit, Epicentre) and then amplified and transcribed into fluorescent cRNA along the entire length of 
the transcripts without $3^{\prime}$ bias utilizing a random priming method. The labeled cRNAs were hybridized to the Human lncRNA Array v2.0 $(8 \times 60 \mathrm{~K}$, Arraystar $)$. After washing the slides, the arrays were scanned using the Agilent Scanner G2505C.

Agilent Feature Extraction software (version 11.0.1.1) was used to analyze the acquired array images. Quantile normalization and subsequent data processing were performed using the GeneSpring GX v11.5.1 software package (Agilent Technologies). After quantile normalization of the raw data, lncRNAs in which all 7 samples displayed flags corresponding to Present or Marginal ("All Targets Value") were selected for further data analysis. The differentially expressed lncRNAs displaying statistical significance between the two groups were identified via Volcano Plot filtering. Finally, hierarchical clustering was performed to elucidate the differentially expressed lncRNA expression profile in the samples.

The experimental protocol was as follows: (1) RNA extraction and RNA QC (described previously); (2) labeling and hybridization (the Agilent Quick Amp Labeling Kit was used for sample labeling and hybridization was performed in Agilent Sure Hyb Hybridization Chambers); (3) data collection and normalization; (4) further data analysis (using Agilent Gene Spring GX v11.5.1 software); and (5) lncRNA classification and subgroup analysis (using homemade scripts). The microarray was performed by KangChen Bio-tech, Shanghai, China.

2.4. Quantitative RT-PCR. Real-time PCR was performed using a LightCycler 480 (Roche, Basel, Switzerland). The reactions were performed in triplicate, and the relative expression of IncRNAs (LOC84740, ENST00000498296, AL359062, and ENST00000438550) was normalized to that of the internal control GAPDH. The primer sequences are presented in Supplementary Table S02.

2.5. Statistics. Statistical analyses were performed using SPSS version 16.0. Receiver operating characteristic (ROC) curve analysis was used to select the threshold expression levels of the lncRNAs detected via QPCR for disease-free survival (DFS). The survival curves were plotted using the KaplanMeier method and were compared using the log-rank test. A multivariate survival analysis was performed using a Cox proportional hazards model (forward). The statistical tests were two-sided, and $P<0.05$ was considered to be significant.

\section{Results}

3.1. Overview of the IncRNA Expression Profiles. Using the IncRNA expression profiles, differentially expressed lncRNAs were determined between the metastatic and primary NPC tumor tissues. The differences in lncRNA expression were evaluated by calculating the normalized fold-change in lncRNA expression between the metastatic/primary tumor $(\mathrm{M} / \mathrm{T})$ samples. The selection criterion was a fold-change threshold of 2.0. A positive fold-change indicated upregulation, whereas a negative fold-change indicated downregulation. Log fold-change corresponded to the $\log 2$ value of the absolute fold-change. Both the fold-change and the $P$ value were normalized. Thousands of lncRNAs were found to be differentially expressed between the metastatic and primary NPC tumors according to UCSC-known gene, Ensemble, RefSeq_NR, H-invDB, NRED, RNAdb, lincRNA, RNAdb, HOX cluster, misc_RNA, UCR, and lncRNAdb.

A total of 33,045 lncRNA probes were used in our lncRNA microarray. Up to 30,610 lncRNAs were detected in all seven samples (Table S03). Thousands of lncRNAs were found to be differentially expressed, and samples in the same group shared many differentially expressed lncRNAs (Figure 1, Table 1, Table S04). A total of 8,088 lncRNAs were identified to be significantly differentially expressed ( $\geq 2$-fold) between the metastatic and primary NPC tumors (Table 1, Table S04). Among these, 3,778 lncRNAs were found to be consistently upregulated; 4,310 lncRNAs were downregulated. Additionally, H19 was found to be 2.2-fold upregulated in the metastatic tissue, which could be related to metastasis (Figure 1, Tables S03-S04). CR620154 (log2 foldchange $\mathrm{M} / \mathrm{T}=94.02$ ) was the most significantly upregulated lncRNA, and TUBA4B ( $\log 2$ fold-change $\mathrm{M} / \mathrm{T}=-1,364.72$ ) was the most significantly downregulated lncRNA (Table 2).

3.2. IncRNA Classification and Subgroup Analysis. According to the function and locus of each lncRNA and its association with protein-encoding RNA, Gibb et al. separated lncRNAs into several categories, such as long intronic ncRNAs, antisense RNAs, and promoter-associated long RNAs [35]. In our microarray study, the lncRNAs were classified into four subgroups: enhancer lncRNAs acting on a nearby coding gene, HOX cluster, lncRNAs near a coding gene, and Rinn lincRNAs [23, 36-38]. The expression levels of the lncRNAs in these subgroups were different between the metastatic and primary NPC tumors (Figure 2, Table 1).

In our study, we found that 477 transcribed regions in HOX loci; of these, 257 were ncRNAs and 220 were HOX coding transcripts (Table S05). In the four randomly paired groups, the number of differentially expressed lncRNAs differed, but several lncRNAs displayed similar changes in expression. Compared with the NPC primary tumors, 70 lncRNAs were found to be differentially expressed in metastatic tissues; 51 coding transcripts were differentially expressed (Table S06, Figure 3(a)). According to the comparative analysis of the four randomly paired groups, $33 \mathrm{lncRNAs}$ were upregulated and $37 \operatorname{lncRNAs}$ were downregulated in the metastatic NPC samples compared with the primary NPC tumor samples (Figure 3(b)). Interestingly, HOTAIR, a known regulatory lncRNA located at the HOX locus, was among the 33 upregulated lncRNAs (Figure 3(c)). HOTAIR has been demonstrated to be an oncogene to modulate the metastasis of breast cancer and NPC [17, 39].

Rinn lincRNAs, a type of lincRNAs identified by Rinn, were also detected in our study [23,38]. A total of 4,199 Rinn lincRNAs were detected in our microarray (Table S07). The number of upregulated and downregulated Rinn lincRNAs 


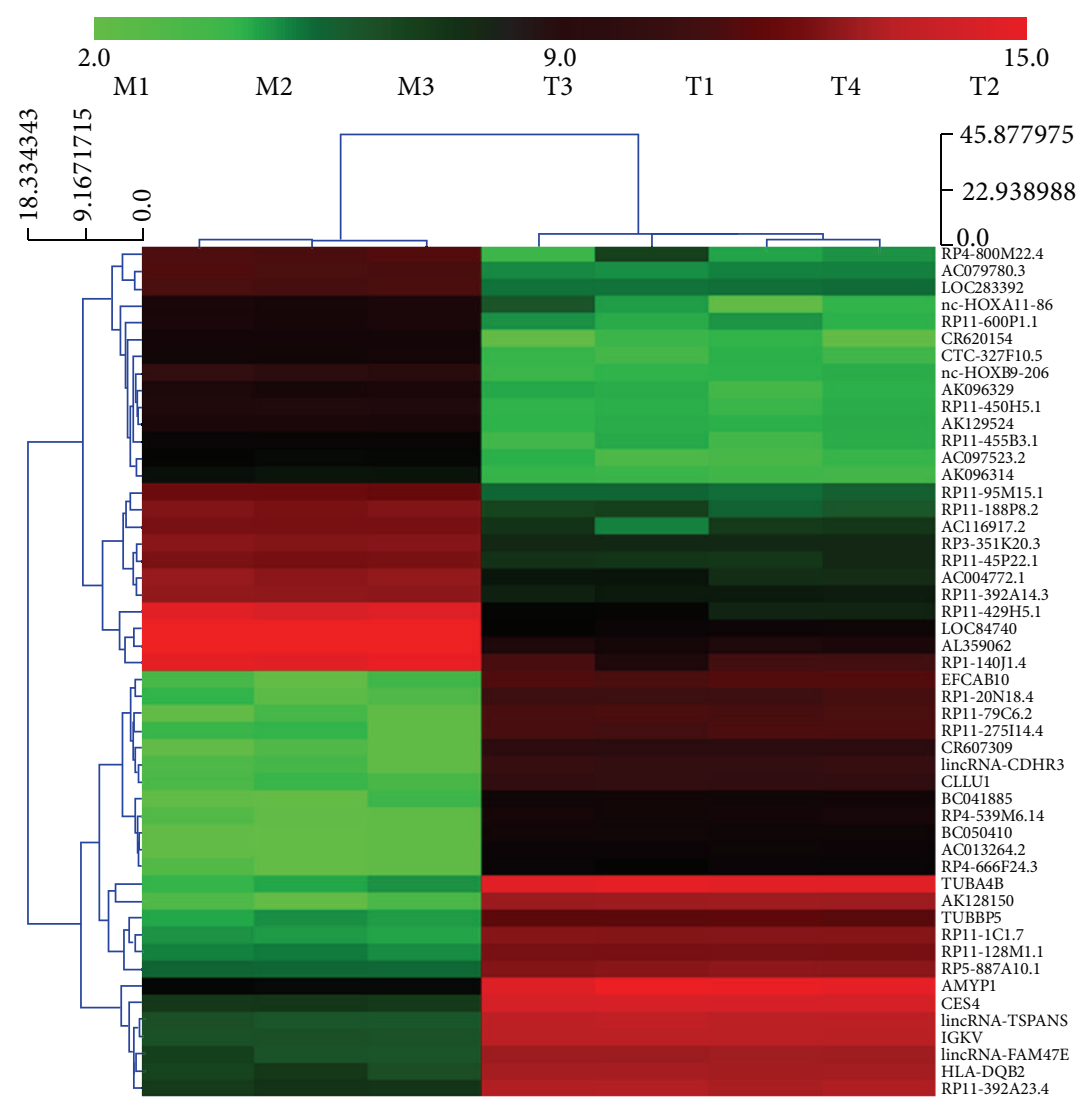

(a)
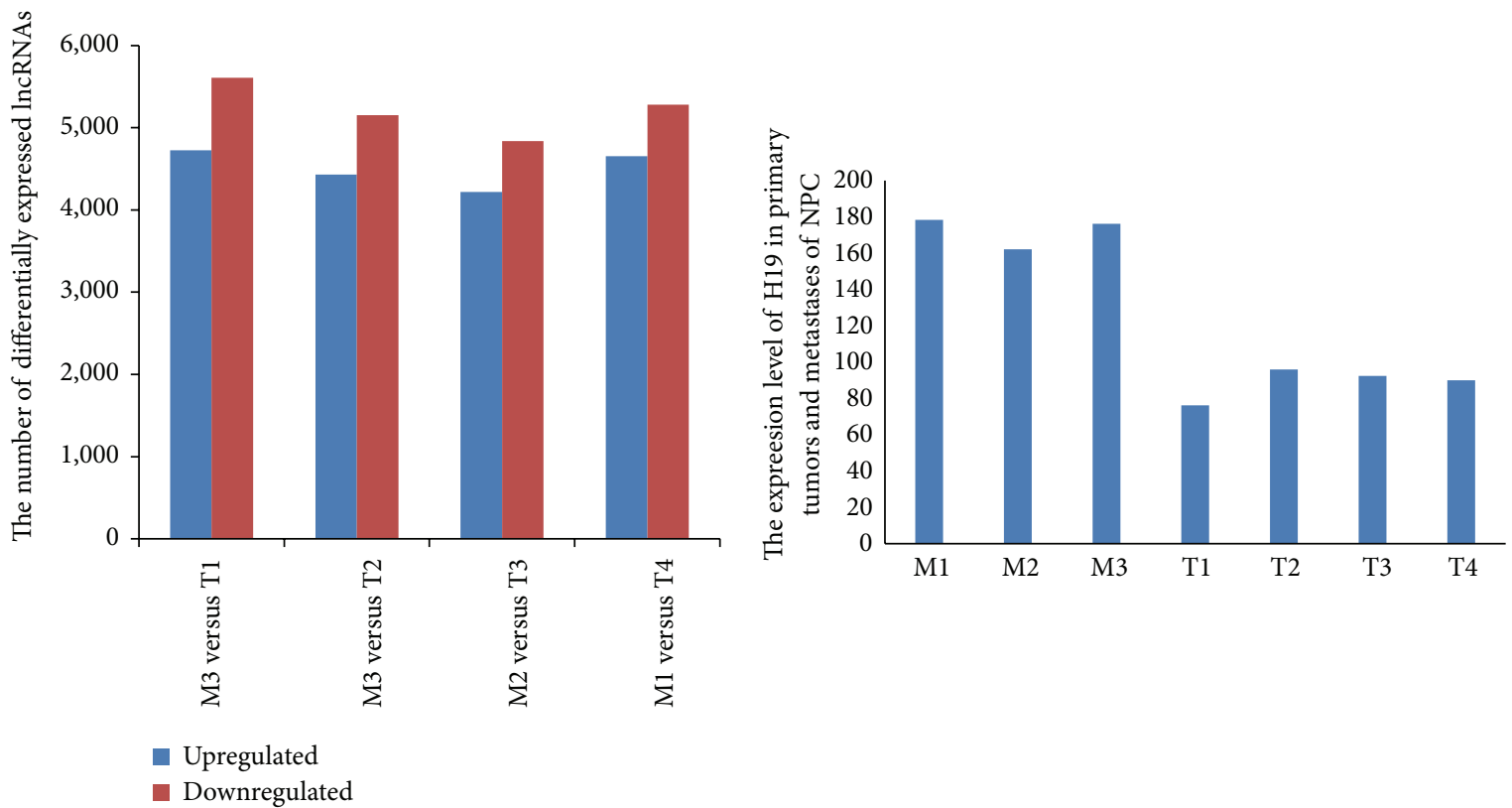

(b)

(c)

FIgURE 1: The number of upregulated and downregulated lncRNAs. (a) Hierarchical clustering was performed based on "All Targets ValuelncRNAs." The results of hierarchical clustering revealed distinct lncRNA expression profiles between the samples. (b) Thousands of lncRNAs were found to be significantly upregulated or downregulated in metastatic NPC tumors compared with primary NPC tumors in seven NPC patients based on microarray analysis. The number of upregulated and downregulated lncRNAs varied between the seven patients. In the four randomly paired $\mathrm{M}$ and T groups, downregulated lncRNAs were more common than upregulated lncRNAs. (c) H19 was found to be upregulated in all metastatic samples $(P<0.001)$; the expression levels of H19 were 1.8 - to 3 -fold higher in the metastatic tumors than in the primary tumors. 
TABLE 1: Summary of the microarray data for the metastatic and primary NPC tumors.

\begin{tabular}{|c|c|c|c|c|c|c|}
\hline \multirow{2}{*}{ Gene type } & \multirow{2}{*}{ RNA expression } & \multicolumn{4}{|c|}{ Fold-change $(n)$} & \multirow{2}{*}{ Differentially expressed RNAs $(n)$} \\
\hline & & $>20$ & $10-20$ & $2-10$ & Total & \\
\hline \multirow{2}{*}{$\operatorname{lncRNA}$} & Upregulated & 65 & 191 & 3,522 & 3,778 & \multirow{2}{*}{8,088} \\
\hline & Downregulated & 198 & 225 & 3,887 & 4,310 & \\
\hline \multirow{2}{*}{$\begin{array}{l}\text { Enhancer lncRNAs regulating a nearby } \\
\text { coding gene }\end{array}$} & Upregulated & 4 & 5 & 94 & 103 & \multirow{2}{*}{462} \\
\hline & Downregulated & 17 & 20 & 322 & 359 & \\
\hline \multirow{2}{*}{ HOX cluster } & Upregulated & 15 & 5 & 51 & 71 & \multirow{2}{*}{121} \\
\hline & Downregulated & 1 & 3 & 46 & 50 & \\
\hline \multirow{2}{*}{$\begin{array}{l}\text { lncRNAs regulating a nearby coding } \\
\text { gene }\end{array}$} & Upregulated & 4 & 5 & 94 & 103 & \multirow{2}{*}{462} \\
\hline & Downregulated & 17 & 20 & 322 & 359 & \\
\hline \multirow{2}{*}{ Rinn lincRNAs } & Upregulated & 6 & 14 & 328 & 348 & \multirow{2}{*}{1,069} \\
\hline & Downregulated & 33 & 28 & 660 & 721 & \\
\hline
\end{tabular}

NPC: nasopharyngeal carcinoma; lncRNA: long noncoding RNA; lincRNA: long intergenic noncoding RNA.

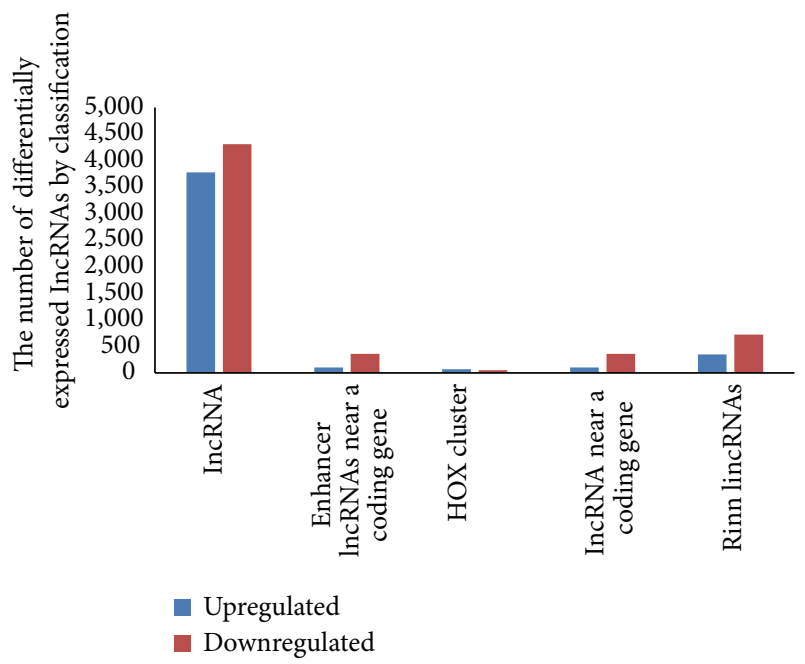

FIGURE 2: The number of upregulated and downregulated lncRNAs in each subgroup. The lncRNAs were classified into four subgroups based on microarray analysis, including enhancer lncRNAs regulating a nearby coding gene, HOX cluster, lincRNAs regulating a nearby coding gene, and Rinn lincRNAs. The number of lncRNAs that were consistently upregulated or downregulated in the metastatic tumors compared with the primary tumors was calculated for each subgroup.

varied between the seven patients. A total of 1,069 Rinn lincRNAs were found to be differentially expressed between the patient samples (Figure 4, Table S08). As shown in Figure 4, the downregulation of the lncRNAs was more common than the upregulation. Among the four paired samples, we found 348 lncRNAs that were consistently upregulated and $721 \mathrm{lncRNAs}$ that were consistently downregulated. The consistently dysregulated lncRNAs in the four groups may function as oncogenes or tumor suppressor genes; this merits further investigation.

Enhancer lncRNAs acting on a nearby coding gene were first found in human cell lines [37]. The present study revealed an unanticipated role of this subgroup of lncRNAs in the
TABLE 2: A collection of significantly differentially expressed lncRNAs detected via microarray analysis in seven NPC patients.

\begin{tabular}{|c|c|c|c|}
\hline \multicolumn{2}{|c|}{ Upregulated } & \multicolumn{2}{|c|}{ Downregulated } \\
\hline $\operatorname{lncRNA}$ & $\begin{array}{c}\log 2 \\
\text { fold-change } \\
(\mathrm{M} / \mathrm{T})\end{array}$ & $\operatorname{lncRNA}$ & $\begin{array}{c}\log 2 \\
\text { fold-change } \\
(\mathrm{M} / \mathrm{T})\end{array}$ \\
\hline CR620154 & 94.02 & TUBA4B & $1,364.72$ \\
\hline LOC84740 & 92.86 & AK128150 & $1,120.20$ \\
\hline nc-HOXB9-206 & 87.55 & RP11-79C6.2 & 303.82 \\
\hline RP4-800M22.4 & 67.25 & EFCAB10 & 274.84 \\
\hline RP11-450H5.1 & 65.75 & RP11-1C1.7 & 208.11 \\
\hline RP11-429H5.1 & 64.15 & RP11-275I14.4 & 206.67 \\
\hline CTC-327F10.5 & 62.57 & CR607309 & 197.51 \\
\hline AK096329 & 58.19 & RP1-20N18.4 & 196.02 \\
\hline AC079780.3 & 55.47 & lincRNA-CDHR3 & 195.01 \\
\hline AK129524 & 53.04 & BC041885 & 141.04 \\
\hline AL359062 & 50.10 & $\begin{array}{l}\text { lincRNA- } \\
\text { TSPAN8 }\end{array}$ & 137.80 \\
\hline RP11-95M15.1 & 46.94 & BC050410 & 131.80 \\
\hline RP11-455B3.1 & 45.48 & RP4-539M6.14 & 130.89 \\
\hline RP11-188P8.2 & 41.74 & IGKV & 125.27 \\
\hline AC097523.2 & 41.72 & AC013264.2 & 122.93 \\
\hline nc-HOXA11-86 & 41.48 & CES4 & 120.27 \\
\hline RP11-600P1.1 & 38.72 & CLLU1 & 108.35 \\
\hline LOC283392 & 33.97 & TUBBP5 & 108.01 \\
\hline AK096314 & 33.87 & RP11-128M1.1 & 105.58 \\
\hline AC116917.2 & 33.61 & RP4-666F24.3 & 100.17 \\
\hline
\end{tabular}

NPC: nasopharyngeal carcinoma; M: metastatic NPC tissue; T: primary NPC tissue.

False discovery rate $($ FDR $)<0.1 \%, P<0.01$.

activation of critical development and differentiation regulators. In this study, many enhancer lncRNAs were found to display increased or decreased expression in M/T. Enhancer lncRNA profiling contained the profiling data of all lncRNAs displaying enhancer-like function (Table S09). A total of 


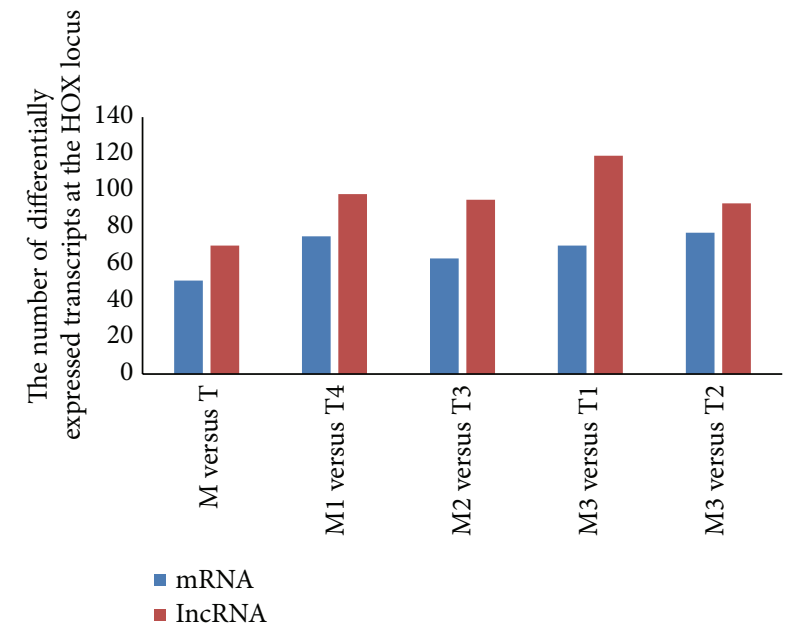

(a)

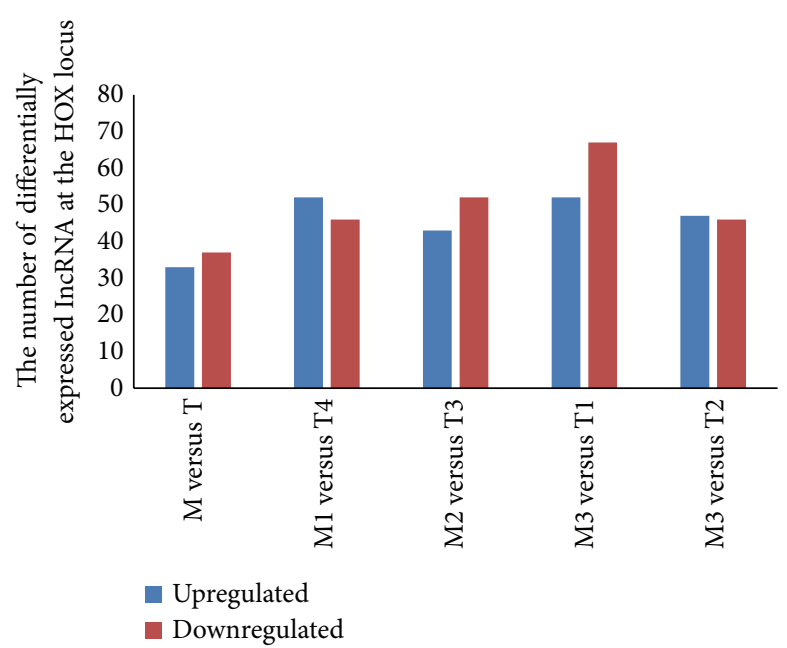

(b)

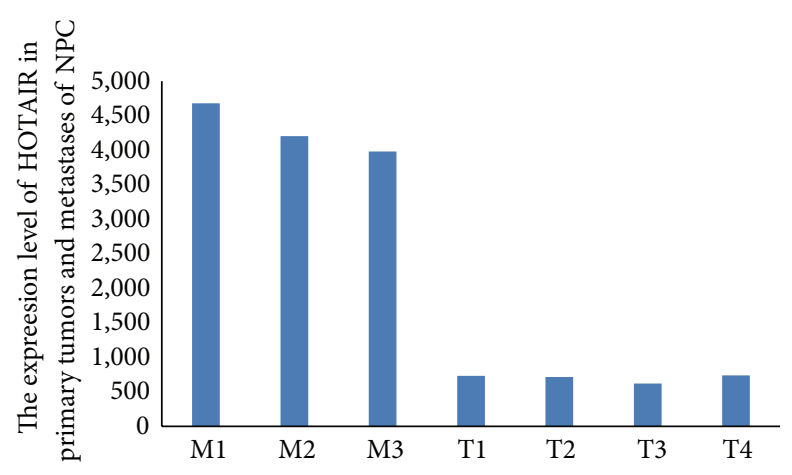

(c)

Figure 3: The number of differentially expressed lncRNAs at the HOX locus. (a) The transcripts at the HOX locus varied between the four randomly paired $\mathrm{M}$ and $\mathrm{T}$ groups. A total of $70 \mathrm{lncRNAs}$ were found to be differentially expressed in the metastatic tissues, and 51 coding transcripts were differentially expressed. (b) Different numbers of lncRNAs were detected in the four randomly paired $\mathrm{M}$ and T groups. A total of $33 \operatorname{lncRNAs}$ at the HOX locus were found to be upregulated in all groups, and $37 \operatorname{lncRNAs}$ were downregulated. (c) HOTAIR was found to be upregulated in the metastatic tumor samples from all of the groups $(P<0.001)$; the expression levels of HOTAIR in the metastatic tumors were 4- to 6-fold higher than those in the primary tumors.

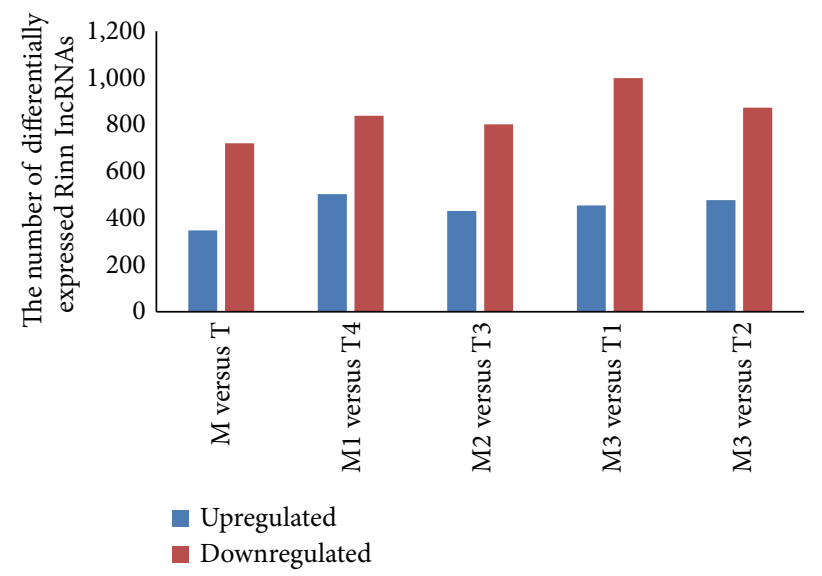

FIGURE 4: The number of differentially expressed Rinn lincRNAs. Rinn lincRNAs are a type of lincRNAs termed based on studies by Rinn. A total of 4,199 Rinn lincRNAs were detected in our microarray analysis. The number of downregulated Rinn lincRNAs was greater than the number of upregulated Rinn lincRNAs. According to the expression levels of all detected Rinn lincRNAs in metastatic and primary NPC tumors, 348 of these lncRNAs displayed consistent upregulation and 721 of these lncRNAs displayed consistent downregulation in the four randomly paired $\mathrm{M}$ and $\mathrm{T}$ groups. 
TABLE 3: The clinicopathological characteristics and their association with the expression levels of four lncRNAs (LOC84740, ENST00000498296, AL359062, and ENST00000438550) in NPC patients.

\begin{tabular}{|c|c|c|c|c|c|c|c|c|c|c|c|c|}
\hline & \multicolumn{3}{|c|}{$\begin{array}{c}\text { LOC } 84740 \\
(n=105)\end{array}$} & \multicolumn{3}{|c|}{$\begin{array}{l}\text { ENST00000498296 } \\
\quad(n=106)\end{array}$} & \multicolumn{3}{|c|}{$\begin{array}{r}\text { AL359062 } \\
(n=106)\end{array}$} & \multicolumn{3}{|c|}{$\begin{array}{l}\text { ENST00000438550 } \\
\quad(n=106)\end{array}$} \\
\hline & $\mathrm{L}$ & $\mathrm{H}$ & $P$ & $\mathrm{~L}$ & $\mathrm{H}$ & $P$ & $\mathrm{~L}$ & $\mathrm{H}$ & $P$ & $\mathrm{~L}$ & $\mathrm{H}$ & $P$ \\
\hline \multicolumn{13}{|l|}{ Age (years) } \\
\hline$<50$ & 53 & 14 & \multirow{2}{*}{0.81} & 25 & 43 & \multirow{2}{*}{0.54} & 34 & 34 & \multirow{2}{*}{0.23} & 12 & 56 & \multirow{2}{*}{0.06} \\
\hline$\geq 50$ & 29 & 9 & & 17 & 21 & & 14 & 24 & & 13 & 25 & \\
\hline \multicolumn{13}{|l|}{ Gender } \\
\hline Male & 66 & 15 & \multirow{2}{*}{0.15} & 32 & 49 & \multirow{2}{*}{1.00} & 37 & 44 & \multirow{2}{*}{0.82} & 11 & 70 & \multirow[t]{2}{*}{0.00} \\
\hline Female & 15 & 8 & & 9 & 15 & & 10 & 14 & & 14 & 10 & \\
\hline \multicolumn{13}{|c|}{ Histological type } \\
\hline $\mathrm{D}$ & 2 & 0 & \multirow{2}{*}{0.00} & 0 & 2 & \multirow{2}{*}{0.52} & 1 & 1 & \multirow{2}{*}{1.00} & 1 & 1 & \multirow{2}{*}{0.42} \\
\hline $\mathrm{U}$ & 80 & 23 & & 42 & 62 & & 47 & 57 & & 24 & 80 & \\
\hline \multicolumn{13}{|c|}{ T classification } \\
\hline T1-2 & 7 & 0 & \multirow{2}{*}{0.34} & 4 & 3 & \multirow{2}{*}{0.43} & 5 & 2 & \multirow{2}{*}{0.24} & 1 & 6 & \multirow{2}{*}{1.00} \\
\hline T3-4 & 75 & 23 & & 38 & 61 & & 43 & 56 & & 24 & 75 & \\
\hline \multicolumn{13}{|c|}{$\mathrm{N}$ classification } \\
\hline N0-1 & 51 & 16 & \multirow{2}{*}{0.63} & 26 & 41 & \multirow{2}{*}{0.84} & 28 & 39 & \multirow{2}{*}{0.42} & 15 & 52 & \multirow{2}{*}{0.81} \\
\hline $\mathrm{N} 2-3$ & 31 & 7 & & 16 & 23 & & 20 & 19 & & 10 & 29 & \\
\hline \multicolumn{13}{|c|}{ Distant metastasis } \\
\hline No & 73 & 18 & 0.18 & 36 & 56 & 0.78 & 44 & 48 & 0.25 & 24 & 68 & 0.18 \\
\hline Yes & 9 & 5 & & 6 & 8 & & 4 & 10 & & 1 & 13 & \\
\hline Local-regic & & & & & & & & & & & & \\
\hline No & 74 & 21 & 1.00 & 40 & 56 & 0.31 & 45 & 51 & 0.34 & 25 & 71 & 0.11 \\
\hline Yes & 8 & 2 & & 2 & 8 & & 3 & 7 & & 0 & 10 & \\
\hline Disease pro & & & & & & & & & & & & \\
\hline No & 65 & 17 & 0.58 & 34 & 49 & 0.64 & 41 & 42 & 0.16 & 24 & 59 & 0.01 \\
\hline Yes & 17 & 6 & & 8 & 15 & & 7 & 16 & 0.10 & 1 & 22 & 0.01 \\
\hline
\end{tabular}

L: low level; H: high level; $P$ : $P$ value; D: differentiated nonkeratinized carcinoma; U: undifferentiated nonkeratinized carcinoma.

1,598 enhancer lncRNAs were detected, of which 468 were differentially expressed. The differentially expressed enhancer lncRNAs and their nearby coding genes (distance $<300 \mathrm{~kb}$ ) are presented in Table S10. As shown in Figure 5, the enhancer lncRNAs were located either upstream or downstream of the coding genes. Some of the enhancer lncRNAs shared the same change in expression with their nearby coding genes, while the others displayed the opposite changes; this was helpful for the identification of functional enhancer lncRNAs.

We performed a further analysis of the lincRNA profiles to identify additional potential regulatory lncRNAs and their target genes among the lincRNAs. The differentially expressed lincRNAs and nearby coding gene pairs (distance $<300 \mathrm{~kb})$ are provided in Table S11 $(P<0.05)$.

3.3. Real-Time Quantitative PCR Validation. Based on this microarray analysis and according to the baseline and foldchange in the expression levels, four different lncRNA members (LOC84740, ENST00000498296, AL359062, and ENST00000438550) were selected to verify their expression levels via QPCR. The results revealed strong consistency among the QPCR results and the microarray data (Figures 6(a)-6(b)). Additionally, the expression levels of the four lncRNAs (LOC84740, ENST00000498296, AL359062, and ENST00000438550) were measured in an independent panel of 106 primary NPC samples via QPCR; however, the sample from one patient did not show expression of LOC84740 (Figure 6(c), Table 3). The clinicopathological characteristics of these 106 patients and the associations between these characteristics and the expression levels of LOC84740, ENST00000498296, AL359062, and ENST00000438550 are presented in Table 3. According to their respective ROC curves, the fold-change cutoff points in the expression thresholds for LOC84740, ENST00000498296, AL359062, and ENST00000438550 were 5.54, 0.37, 3.76, and 0.43, respectively. The expression levels of the lncRNAs were categorized into high and low levels accordingly. A high ENST00000438550 expression level was associated with disease progression among NPC patients $(P=0.01)$.

\subsection{Prognosis of NPC Patients Displaying Differentially} Expressed lncRNAs. To further confirm the prognostic value of these lncRNAs for NPC, the DFS of the four lncRNAs was analyzed. Among them, only ENST00000438550 was a 


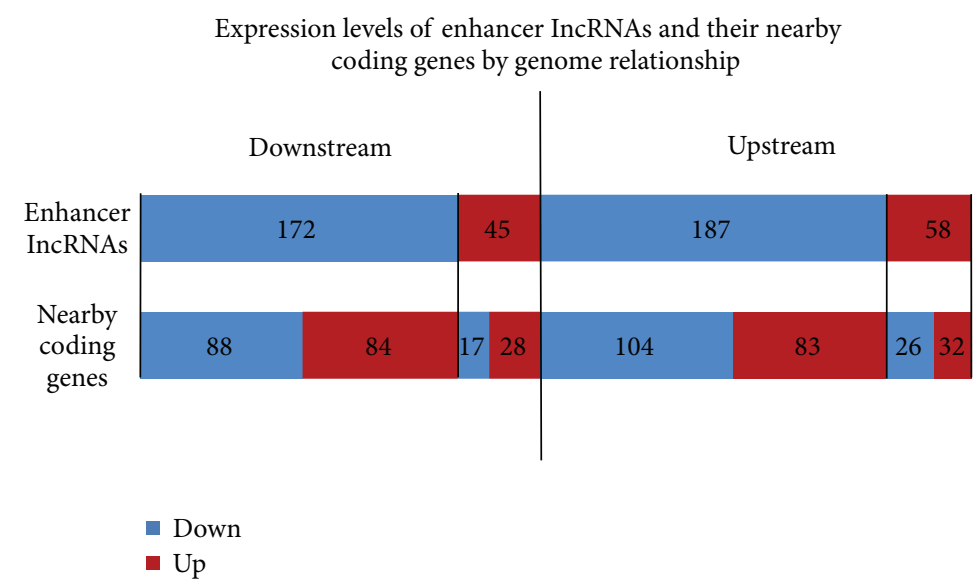

FIGURE 5: Expression levels of enhancer lncRNAs and their nearby coding genes based on genome relationship analysis. A total of 245 enhancer lncRNAs upstream of their nearby coding genes were differentially expressed; 58 of these enhancer lncRNAs were upregulated and 187 were downregulated. Additionally, 217 enhancer lncRNAs downstream of their nearby coding genes were differentially expressed; 45 of these enhancer lncRNAs were upregulated and 172 were downregulated. Some of the nearby coding genes displayed consistent upregulation or downregulation in concert with that of their corresponding enhancer lncRNAs, whereas other nearby coding genes displayed opposite differences.

significant predictor of disease progression in NPC patients (3-year DFS of $96 \%$ and $73 \%$ for the low and high level groups, respectively, $P=0.02$, Figure 7). A multivariate analysis was performed using the COX proportional hazards model to analyze the prognostic values of age, gender, $\mathrm{T}$ classification, $\mathrm{N}$ classification, and the expression levels of LOC84740, ENST00000498296, AL359062, and ENST00000438550. The results revealed that only the expression level of ENST00000438550 was an independent prognostic indicator of disease progression in NPC patients (Chi square $=6.64, P=0.01$ ). These results suggested that ENST00000438550 could serve as a prognostic marker in NPC patients.

\section{Discussion}

The present study was the first to demonstrate that lncRNAs are differentially expressed between metastatic and primary NPC tumors. There have been no previous reports describing lncRNA expression profiles of NPC samples that also performed a differential expression analysis. Furthermore, this study was the first to demonstrate that a high expression level of ENST00000438550 is an independent indicator of disease progression in NPC patients.

Epstein-Barr virus (EBV) plays very important roles in the carcinogenesis of NPC. EBV exhibits tumorigenic potential due to a unique set of latent genes. Latent membrane protein-1 (LMP1) is the principal oncogene, and its expression level is a prognostic marker of NPC [40]. With the development of microarray technology, novel potential therapeutic targets as well as diagnostic and prognostic biomarkers have been identified based on gene expression array analyses. lncRNA expression array analysis has been used in oncology studies in recent years. A variety of lncRNAs, including ANRIL, MEG3 and HULC, either promote or suppress the development of cancer [41-44]. Among these, XIST is a well-known imprinted lncRNA that is abnormally expressed in ovarian and breast cancers $[45,46]$. MALAT- 1 was the first IncRNA that was found to be associated with high metastatic potential and poor patient prognosis in non-smallcell lung cancer patients [47]. MALAT-1 is also upregulated in other human cancers, such as breast cancer, prostate cancer, colorectal cancer, liver cancer, and uterine cancer [48-51]. These findings imply an association between lncRNAs and carcinogenesis.

The altered expression of many genes has been reported to be associated with the development of NPC $[52,53]$. HOTAIR, a lincRNA in the mammalian HOXC locus, was the first lincRNA that was found to be systematically dysregulated during breast cancer progression via microarray analysis [17]. Further evidence indicates that HOTAIR reprograms the chromatin state to promote cancer metastasis and primary tumor growth in vivo [17]. HOTAIR has been proposed as a putative biomarker for metastasis of human malignant tumors, and it is a powerful predictor of eventual metastasis and death $[17,29]$. HOTAIR is aberrantly expressed in several carcinomas, including NPC [29, 39, 54-56]. HOTAIR is upregulated in cases of NPC at more advanced clinical stage and with increased lymph node tumor burden [29]. In our study, HOTAIR was also consistently upregulated in metastatic samples, indicating that increased HOTAIR expression is associated with the progression and development of NPC. H19, another imprinted lncRNA gene with high expression levels during vertebrate embryo development, is downregulated in most tissues shortly after birth [57]. Its loss of imprinting and aberrant expression has been detected in various cancers and has been demonstrated to play a key role in oncogenesis and tumor suppression [18, 32, 58-61]. H19 expression is induced by hypermethylation of its promoter region. H19 is significantly upregulated in the undifferentiated human NPC cell line CNE-2, but it is not expressed in well-differentiated human HK1 NPC cells [32]. 


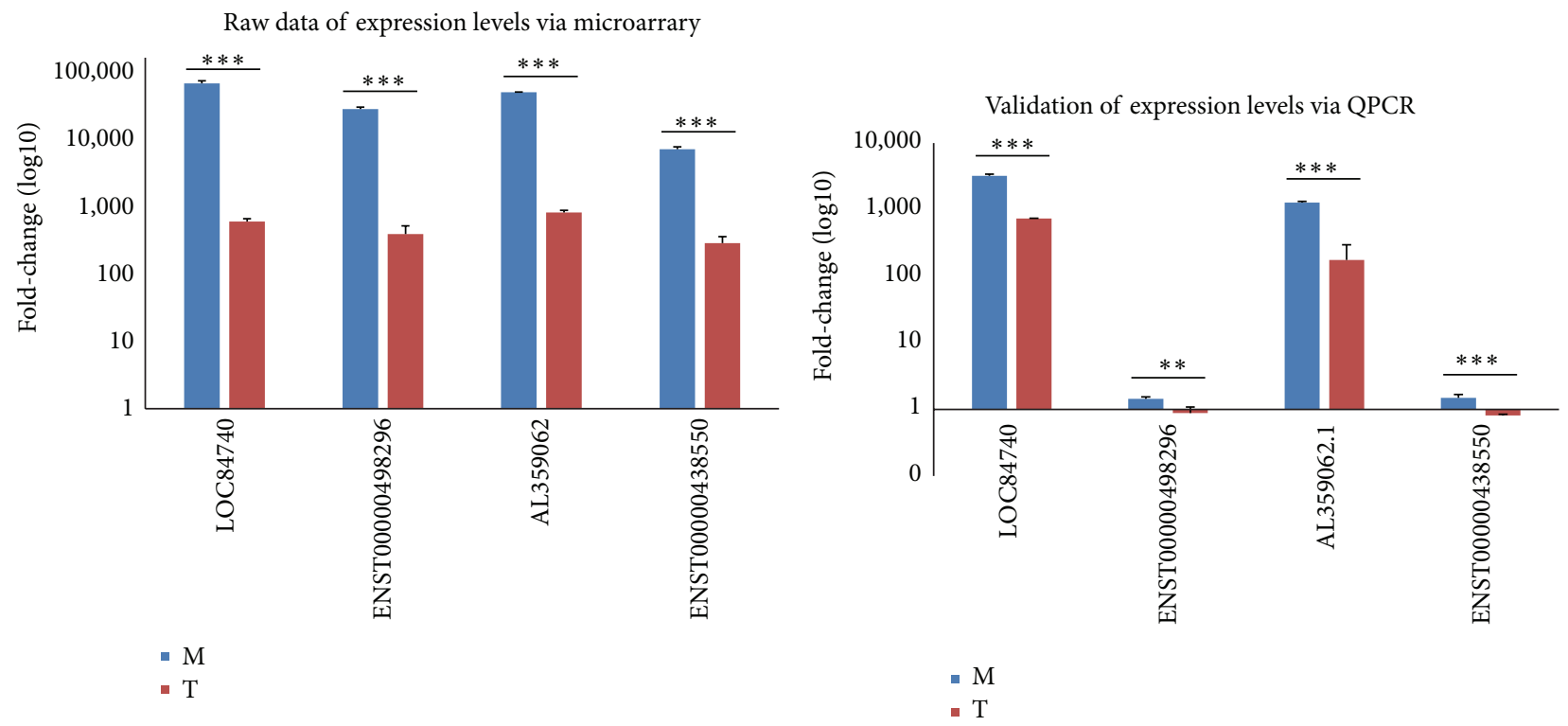

(a)

(b)

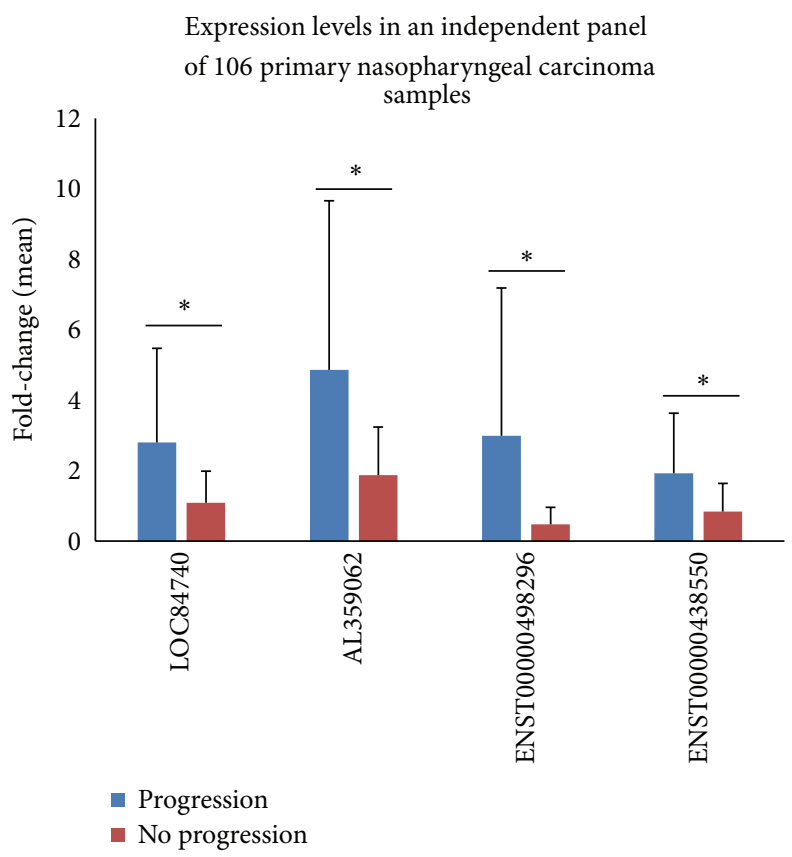

(c)

Figure 6: Real-time quantitative PCR validation. (a) Raw data of the expression levels of the four lncRNAs (LOC84740, ENST00000498296, AL359062, and ENST00000438550) based on microarray analysis. All four of these lncRNAs displayed a high basal expression level in metastatic and primary NPC tumors, but their expression levels significantly differed between the two groups. ${ }^{* * *} P<0.001$. (b) Validation of the microarray data. All four lncRNAs (LOC84740, ENST00000498296, AL359062, and ENST00000438550) were differentially expressed in the metastatic and primary NPC tumors based on microarray analysis, which was validated via QPCR using the same tissues. The validation results of the four lncRNAs indicated that the microarray data strongly correlated with the QPCR results. ${ }^{* * *} P<0.001$ and ${ }^{* *} P<0.01$. (c) The expression levels of four lncRNAs (LOC84740, ENST00000498296, AL359062, and ENST00000438550) were measured in an independent panel of 106 primary NPC samples via QPCR. These lncRNAs displayed higher expression levels in NPC primary tumors with progression than in those without progression. ${ }^{*} P<0.05$.

Our study observed that H19 expression was upregulated in metastatic NPC tumors compared with primary NPC tumors; this result suggests that $\mathrm{H} 19$ expression is related to NPC progression.
lincRNA LINC00312 is significantly downregulated in NPC tissues compared with noncancerous nasopharyngeal epithelial tissues as assessed by a NPC tissue microarray [33]. However, we did not find LINC00312 in our differentially 


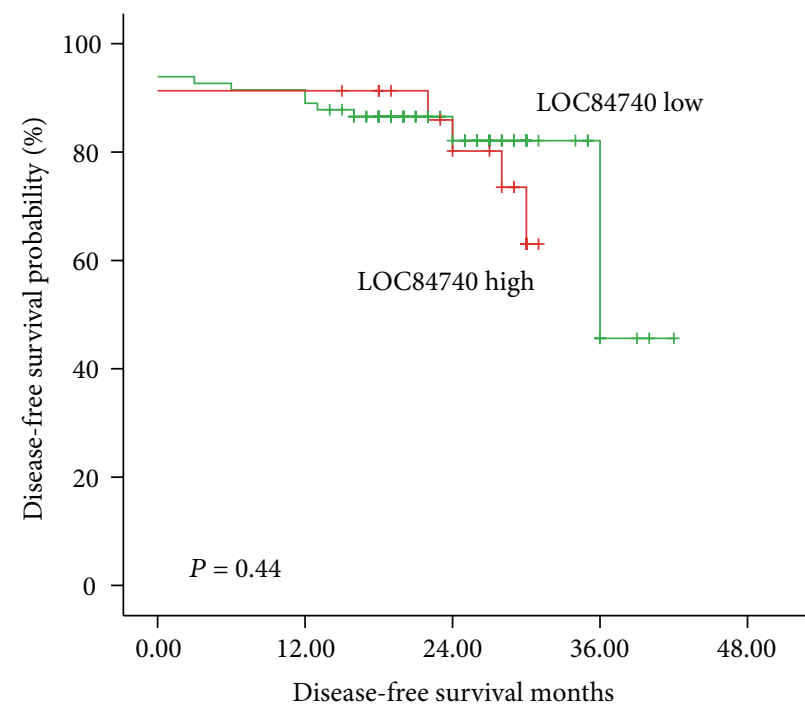

(a)

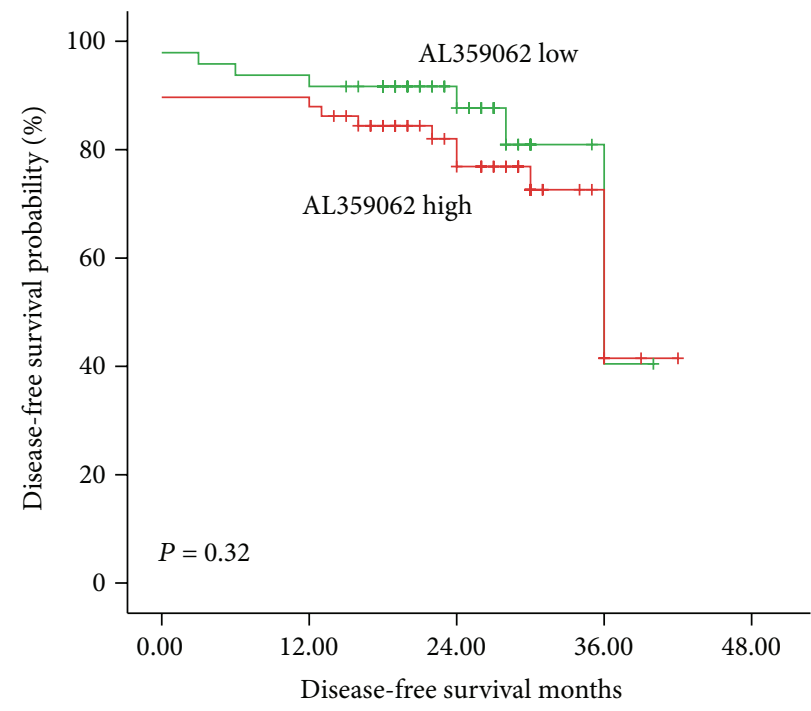

(c)

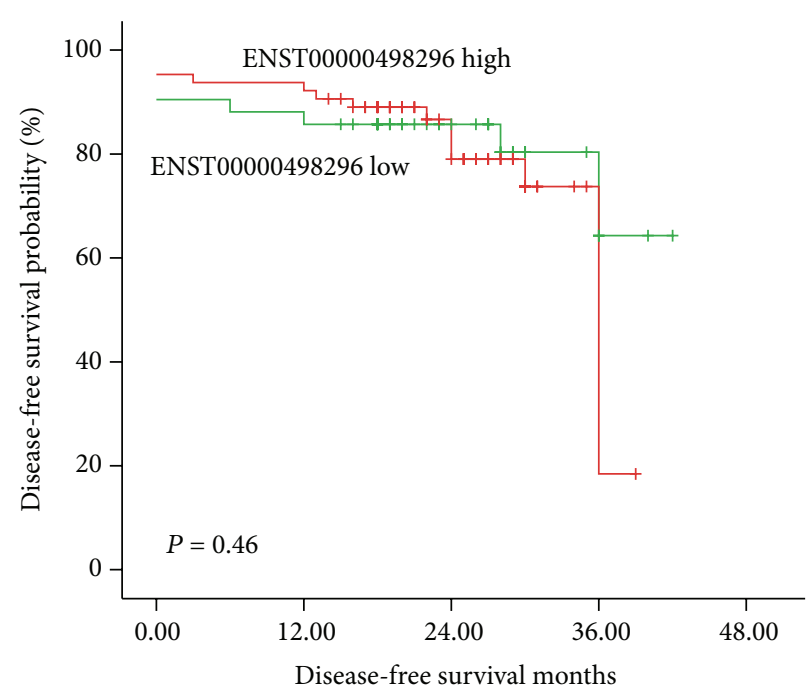

(b)

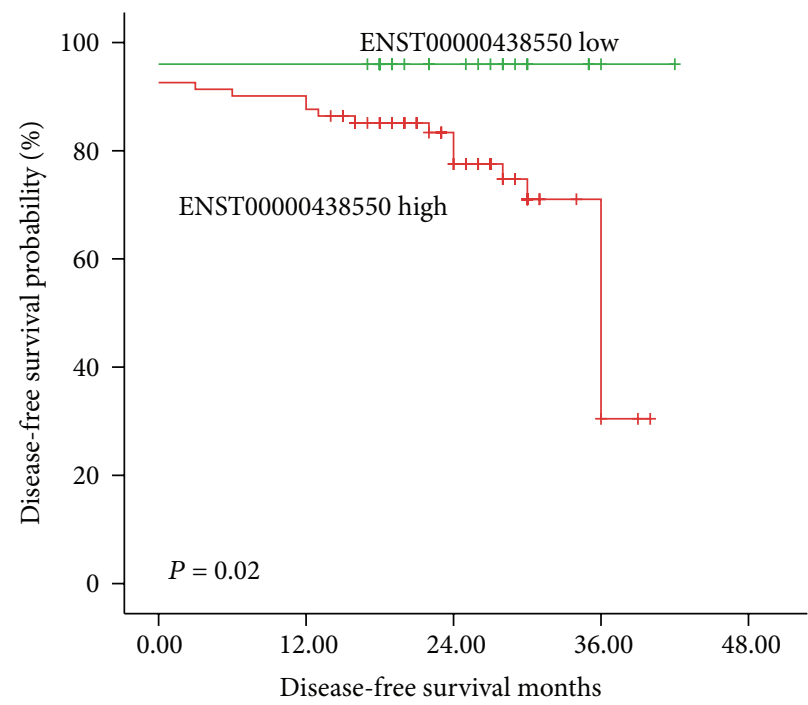

(d)

Figure 7: DFS. The expression level of ENST00000438550 correlated with disease progression in NPC patients; those displaying a high ENST00000438550 expression level experienced a significantly shorter DFS (d). However, the expression levels of LOC84740, ENST00000498296, and AL359062 were not correlated with the DFS of the NPC patients ((a)-(c)).

expressed lncRNAs. The expression of LINC00312 decreased with NPC progression. In addition, only half of the NPC samples express LINC00312, and the number of samples used in our microarray study is limited. Five lncRNAs (lnc-C22orf32-1, lnc-TLR4-1, lnc-BCL2L11-3, lnc-AL355149.11 , and lnc-ZNF674-1) were differentially expressed in NPC compared with normal nasopharyngeal epithelial tissues in the microarray data set GSE12452 [34]. Four of these lncRNAs (lnc-C22orf32-1, lncTLR4-1, lnc-AL355149.1-1, and lnc-ZNF674-1) demonstrated significant expression differences between primary NPC and normal nasopharyngeal samples via QPCR. Only lnc-BCL2L11-3 was upregulated in the recurrent NPC tissues compared with the paired normal tissues. lnc-AL355149.1-1 and lnc-ZNF674-1 were downregulated compared with primary NPC. Unfortunately, none of the five lncRNAs were identified among the differentially expressed lncRNAs based on our microarray data. Our research focuses on NPC metastasis, and the expression of the five lncRNAs varied during carcinogenesis and recurrence.

Based on this microarray analysis and according to the baseline and fold-change in the expression levels, four IncRNAs (LOC84740, ENST00000498296, AL359062, and ENST00000438550) were selected to validate the microarray results and to evaluate their roles as biomarkers in NPC patients. Consistent with the microarray results, the four IncRNAs were differentially expressed based on QPCR. To 
further illustrate the relationship between these four lncRNAs and NPC prognosis, we analyzed the expression levels of these four lncRNAs via QPCR and evaluated their potential values as prognostic indicators of NPC. We found that, among these four lncRNAs, only ENST00000438550 was an independent prognostic indicator of disease progression in NPC patients. The expression level of ENST00000438550 was negatively correlated with the prognosis of NPC patients; this suggests that elucidating the role of ENST00000438550 in NPC progression may contribute to understanding of the mechanism of NPC metastasis.

\section{Conclusion}

To the best of our knowledge, few differentially expressed lncRNAs have been reported in NPC, and this is the first report elucidating the lncRNA expression profiles of metastatic and primary NPC tumor tissues. Further investigation is required in the search for additional functional lncRNAs in NPC. This study has limitations, including the limited sample number for microarray analysis, which was partially due to the difficulty in conducting bone metastases biopsies. In brief, our finding provides new insights into understanding NPC. lncRNAs may underlie novel mechanisms of NPC and may represent potential targets for NPC treatment and prognostic factors for NPC, which are expected to be elucidated in the near future.

\section{Conflict of Interests}

The authors declare that there is no conflict of interests regarding the publication of this study.

\section{Authors' Contribution}

Wei Zhang and Lin Wang contributed equally to this paper.

\section{Acknowledgments}

This work was supported by grants from 973 Projects (2010CB912800, 2011CB504203) from Ministry of Science and Technology of China, the National Nature Science Foundation of China (81172041, 81472525, 81372819, 81230060, and 81261140373), the Specialized Research Fund for the Doctoral Program of Higher Education (20120171110075), Science Foundation of Guangdong Province (S2012030006287), the Guangzhou Science and Technology Bureau (2014J4100170), and Sun Yat-Sen University (13ykzd14).

\section{References}

[1] K. Shanmugaratnam, "Nasopharyngeal carcinoma: epidemiology, histopathology and aetiology," Annals of the Academy of Medicine Singapore, vol. 9, no. 3, pp. 289-295, 1980.

[2] M. C. Yu and J.-M. Yuan, "Epidemiology of nasopharyngeal carcinoma," Seminars in Cancer Biology, vol. 12, no. 6, pp. 421429, 2002.

[3] S.-H. Ou, J. A. Zell, A. Ziogas, and H. Anton-Culver, "Epidemiology of nasopharyngeal carcinoma in the United States: improved survival of Chinese patients within the keratinizing squamous cell carcinoma histology," Annals of Oncology, vol. 18, no. 1, pp. 29-35, 2007.

[4] Y. S. Zong, R. F. Zhang, S. Y. He, and H. Qiu, "Histopathologic types and incidence of malignant nasopharyngeal tumors in Zhongshan county," Chinese Medical Journal, vol. 96, no. 7, pp. 511-516, 1983.

[5] T. L. Vaughan, J. A. Shapiro, R. D. Burt et al., "Nasopharyngeal cancer in a low-risk population: defining risk factors by histological type," Cancer Epidemiology Biomarkers \& Prevention, vol. 5 , no. 8, pp. 587-593, 1996.

[6] A. Mutirangura, W. Pornthanakasem, A. Theamboonlers et al., "Epstein-Barr viral DNA in serum of patients with nasopharyngeal carcinoma," Clinical Cancer Research, vol. 4, no. 3, pp. 665669, 1998.

[7] J.-C. Lin, K. Y. Chen, W.-Y. Wang et al., "Detection of EpsteinBarr virus DNA in the peripheral-blood cells of patients with nasopharyngeal carcinoma: relationship to distant metastasis and survival," Journal of Clinical Oncology, vol. 19, no. 10, pp. 2607-2615, 2001

[8] J. C. Lin, W. Y. Wang, K. Y. Chen et al., "Quantification of plasma Epstein-Barr virus DNA in patients with advanced nasopharyngeal carcinoma," The New England Journal of Medicine, vol. 350, pp. 2461-2470, 2004.

[9] E.-L. Tan, S.-C. Peh, and C.-K. Sam, "Analyses of Epstein-Barr virus latent membrane protein-1 in Malaysian nasopharyngeal carcinoma: high prevalence of 30-bp deletion, Xhol polymorphism and evidence of dual infections," Journal of Medical Virology, vol. 69, no. 2, pp. 251-257, 2003.

[10] W.-H. Jia, X.-Y. Luo, B.-J. Feng et al., "Traditional Cantonese diet and nasopharyngeal carcinoma risk: a large-scale case-control study in Guangdong, China," BMC Cancer, vol. 10, article 446, 2010.

[11] E. E. Vokes, D. N. Liebowitz, and R. R. Weichselbaum, “Nasopharyngeal carcinoma," The Lancet, vol. 350, no. 9084, pp. 1087-1091, 1997.

[12] J. Polesel, S. Franceschi, R. Talamini et al., “Tobacco smoking, alcohol drinking, and the risk of different histological types of nasopharyngeal cancer in a low-risk population," Oral Oncology, vol. 47, no. 6, pp. 541-545, 2011.

[13] A. W. M. Lee, Y. F. Poon, W. Foo et al., "Retrospective analysis of 5037 patients with nasopharyngeal carcinoma treated during 1976-1985: overall survival and patterns of failure," International Journal of Radiation Oncology Biology Physics, vol. 23, no. 2, pp. 261-270, 1992.

[14] J. S. Mattick, “The genetic signatures of noncoding RNAs," PLoS Genetics, vol. 5, Article ID e1000459, 2009.

[15] K. V. Prasanth and D. L. Spector, "Eukaryotic regulatory RNAs: an answer to the "genome complexity" conundrum," Genes \& Development, vol. 21, no. 1, pp. 11-42, 2007.

[16] J. S. Mattick and I. V. Makunin, "Non-coding RNA," Human Molecular Genetics, vol. 15, pp. R17-R29, 2006.

[17] M. Chaudhary, S. Gupta, S. Khare, and S. Lal, "Diagnosis of tuberculosis in an era of HIV pandemic: a review of current status and future prospects," Indian Journal of Medical Microbiology, vol. 28, no. 4, pp. 281-289, 2010.

[18] A. Gabory, M.-A. Ripoche, T. Yoshimizu, and L. Dandolo, "The H19 gene: regulation and function of a non-coding RNA," Cytogenetic and Genome Research, vol. 113, no. 1-4, pp. 188-193, 2006. 
[19] S. Lottin, E. Adriaenssens, T. Dupressoir et al., "Overexpression of an ectopic $\mathrm{H} 19$ gene enhances the tumorigenic properties of breast cancer cells," Carcinogenesis, vol. 23, no. 11, pp. 1885-1895, 2002.

[20] J. E. Wilusz, H. Sunwoo, and D. L. Spector, "Long noncoding RNAs: functional surprises from the RNA world," Genes \& Development, vol. 23, no. 13, pp. 1494-1504, 2009.

[21] P. P. Amaral and J. S. Mattick, "Noncoding RNA in development," Mammalian Genome, vol. 19, no. 7-8, pp. 454-492, 2008.

[22] M. E. Dinger, P. P. Amaral, T. R. Mercer, and J. S. Mattick, "Pervasive transcription of the eukaryotic genome: functional indices and conceptual implications," Briefings in Functional Genomics and Proteomics, vol. 8, no. 6, Article ID elp038, pp. 407-423, 2009.

[23] M. Guttman, I. Amit, M. Garber et al., "Chromatin signature reveals over a thousand highly conserved large non-coding RNAs in mammals," Nature, vol. 458, no. 7235, pp. 223-227, 2009.

[24] T. R. Mercer, M. E. Dinger, and J. S. Mattick, "Long non-coding RNAs: insights into functions," Nature Reviews Genetics, vol. 10, no. 3, pp. 155-159, 2009.

[25] J.Zhao, B. K. Sun, J. A. Erwin, J.-J. Song, and J. T. Lee, "Polycomb proteins targeted by a short repeat RNA to the mouse X chromosome," Science, vol. 322, no. 5902, pp. 750-756, 2008.

[26] O. Wapinski and H. Y. Chang, "Long noncoding RNAs and human disease," Trends in Cell Biology, vol. 21, no. 6, pp. 354361, 2011.

[27] M. Huarte and J. L. Rinn, "Large non-coding RNAs: missing links in cancer?” Human Molecular Genetics, vol. 19, no. 2, pp. R152-R161, 2010.

[28] K. L. Yap, S. Li, A. M. Muñoz-Cabello et al., "Molecular interplay of the noncoding RNA ANRIL and methylated histone H3 lysine 27 by polycomb CBX7 in transcriptional silencing of INK4a," Molecular Cell, vol. 38, no. 5, pp. 662-674, 2010.

[29] Y. Nie, X. Liu, S. Qu, E. Song, H. Zou, and C. Gong, "Long noncoding RNA HOTAIR is an independent prognostic marker for nasopharyngeal carcinoma progression and survival," Cancer Science, vol. 104, no. 4, pp. 458-464, 2013.

[30] V. Tripathi, J. D. Ellis, Z. Shen et al., "The nuclear-retained noncoding RNA MALAT1 regulates alternative splicing by modulating SR splicing factor phosphorylation," Molecular Cell, vol. 39, no. 6, pp. 925-938, 2010.

[31] T. Hung, Y. Wang, M. F. Lin et al., "Extensive and coordinated transcription of noncoding RNAs within cell-cycle promoters," Nature Genetics, vol. 43, no. 7, pp. 621-629, 2011.

[32] A. Ng, J. P. Tang, C. H. K. Goh, and K. M. Hui, "Regulation of the H19 imprinting gene expression in human nasopharyngeal carcinoma by methylation," International Journal of Cancer, vol. 104, no. 2, pp. 179-187, 2003.

[33] W. Zhang, C. Huang, Z. Gong et al., "Expression of LINC00312, a long intergenic non-coding RNA, is negatively correlated with tumor size but positively correlated with lymph node metastasis in nasopharyngeal carcinoma," Journal of Molecular Histology, vol. 44, pp. 545-554, 2013.

[34] W. Gao, J. Y. Chan, and T.-S. Wong, "Differential expression of long noncoding RNA in primary and recurrent nasopharyngeal carcinoma," BioMed Research International, vol. 2014, Article ID 404567, 9 pages, 2014.

[35] E. A. Gibb, C. J. Brown, and W. L. Lam, "The functional role of long non-coding RNA in human carcinomas," Molecular Cancer, vol. 10, article 38, 2011.
[36] J. L. Rinn, M. Kertesz, J. K. Wang et al., "Functional demarcation of active and silent chromatin domains in human HOX loci by noncoding RNAs," Cell, vol. 129, no. 7, pp. 1311-1323, 2007.

[37] U. A. Ørom, T. Derrien, M. Beringer et al., "Long noncoding RNAs with enhancer-like function in human cells," Cell, vol. 143, no. 1, pp. 46-58, 2010.

[38] A. M. Khalil, M. Guttman, M. Huarte et al., "Many human large intergenic noncoding RNAs associate with chromatinmodifying complexes and affect gene expression," Proceedings of the National Academy of Sciences of the United States of America, vol. 106, no. 28, pp. 11667-11672, 2009.

[39] T. Chiyomaru, S. Yamamura, S. Fukuhara et al., "Genistein inhibits prostate cancer cell growth by targeting miR-34a and oncogenic HOTAIR," PLoS ONE, vol. 8, Article ID e70372, 2013.

[40] J. Chou, Y.-C. Lin, J. Kim et al., "Nasopharyngeal carcinomareview of the molecular mechanisms of tumorigenesis," Head and Neck, vol. 30, no. 7, pp. 946-963, 2008.

[41] K. Panzitt, M. M. O. Tschernatsch, C. Guelly et al., "Characterization of HULC, a novel gene with striking up-regulation in hepatocellular carcinoma, as noncoding RNA," Gastroenterology, vol. 132, no. 1, pp. 330-342, 2007.

[42] X. Zhang, Y. Zhou, K. R. Mehta et al., "A pituitary-derived MEG3 isoform functions as a growth suppressor in tumor cells," Journal of Clinical Endocrinology \& Metabolism, vol. 88, no. 11, pp. 5119-5126, 2003.

[43] W. Yu, D. Gius, P. Onyango et al., "Epigenetic silencing of tumour suppressor gene p15 by its antisense RNA," Nature, vol. 451, no. 7175, pp. 202-206, 2008.

[44] N. Popov and J. Gil, "Epigenetic regulation of the INK4B-ARFINK4 $a$ locus: in sickness and in health," Epigenetics, vol. 5, no. 8, pp. 685-690, 2010.

[45] K.-C. Huang, P. H. Rao, C. C. Lau et al., "Relationship of XIST expression and responses of ovarian cancer to chemotherapy," Molecular Cancer Therapeutics, vol. 1, no. 10, pp. 769-776, 2002.

[46] S. M. Sirchia, S. Tabano, L. Monti et al., "Misbehaviour of XIST RNA in breast cancer cells," PLoS ONE, vol. 4, Article ID e5559, 2009.

[47] P. Ji, S. Diederichs, W. Wang et al., "MALAT-1, a novel noncoding RNA, and thymosin $\beta 4$ predict metastasis and survival in early-stage non-small cell lung cancer," Oncogene, vol. 22, no. 39, pp. 8031-8041, 2003.

[48] A. Guffanti, M. Iacono, P. Pelucchi et al., "A transcriptional sketch of a primary human breast cancer by 454 deep sequencing," BMC Genomics, vol. 10, article 163, 2009.

[49] K. Yamada, J. Kano, H. Tsunoda et al., "Phenotypic characterization of endometrial stromal sarcoma of the uterus," Cancer Science, vol. 97, no. 2, pp. 106-112, 2006.

[50] R. Lin, S. Maeda, C. Liu, M. Karin, and T. S. Edgington, "A large noncoding RNA is a marker for murine hepatocellular carcinomas and a spectrum of human carcinomas," Oncogene, vol. 26, no. 6, pp. 851-858, 2007.

[51] J.-H. Luo, B. Ren, S. Keryanov et al., "Transcriptomic and genomic analysis of human hepatocellular carcinomas and hepatoblastomas," Hepatology, vol. 44, no. 4, pp. 1012-1024, 2006.

[52] J. Lu, G. Getz, E. A. Miska et al., "MicroRNA expression profiles classify human cancers," Nature, vol. 435, no. 7043, pp. 834-838, 2005.

[53] S.-L. Yu, H.-Y. Chen, G.-C. Chang et al., "MicroRNA signature predicts survival and relapse in lung cancer," Cancer Cell, vol. 13, no. 1, pp. 48-57, 2008. 
[54] C. J. Woo and R. E. Kingston, "HOTAIR lifts noncoding RNAs to new levels," Cell, vol. 129, no. 7, pp. 1257-1259, 2007.

[55] K. Kim, I. Jutooru, G. Chadalapaka et al., "HOTAIR is a negative prognostic factor and exhibits pro-oncogenic activity in pancreatic cancer," Oncogene, vol. 32, no. 13, pp. 1616-1625, 2013.

[56] X.-B. Lv, G.-Y. Lian, H.-R. Wang, E. Song, H. Yao, and M.H. Wang, "Long noncoding RNA HOTAIR is a prognostic marker for esophageal squamous cell carcinoma progression and survival," PLoS ONE, vol. 8, Article ID e63516, 2013.

[57] O. Lustig, I. Ariel, J. Ilan, E. Lev-Lehman, N. de-Groot, and A. Hochberg, "Expression of the imprinted gene H19 in the human fetus," Molecular Reproduction and Development, vol. 38, no. 3, pp. 239-246, 1994.

[58] I. Ariel, N. de Groot, and A. Hochberg, "Imprinted H19 gene expression in embryogenesis and human cancer: the oncofetal connection," The American Journal of Medical Genetics, vol. 91, pp. 46-50, 2000.

[59] K. Hashimoto, C. Azuma, Y. Tokugawa et al., "Loss of H19 imprinting and up-regulation of $H 19$ and SNRPN in a case with malignant mixed müllerian tumor of the uterus," Human Pathology, vol. 28, no. 7, pp. 862-865, 1997.

[60] K. Hibi, H. Nakamura, A. Hirai et al., "Loss of H19 imprinting in esophageal cancer," Cancer Research, vol. 56, no. 3, pp. 480-482, 1996.

[61] Y. Fellig, I. Ariel, P. Ohana et al., "H19 expression in hepatic metastases from a range of human carcinomas," Journal of Clinical Pathology, vol. 58, no. 10, pp. 1064-1068, 2005. 


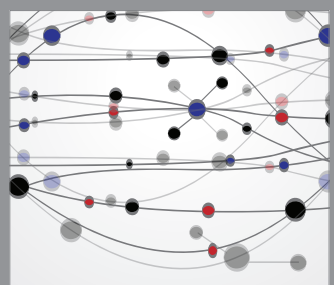

The Scientific World Journal
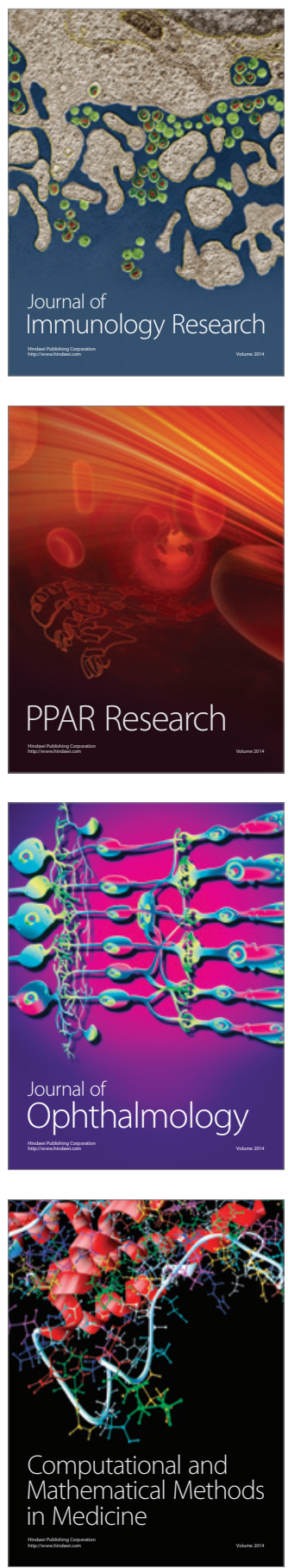

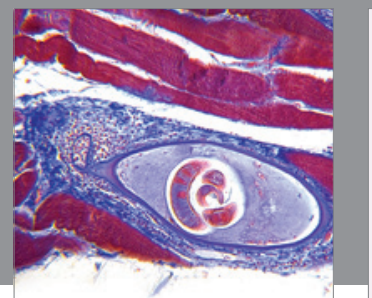

Gastroenterology

Research and Practice
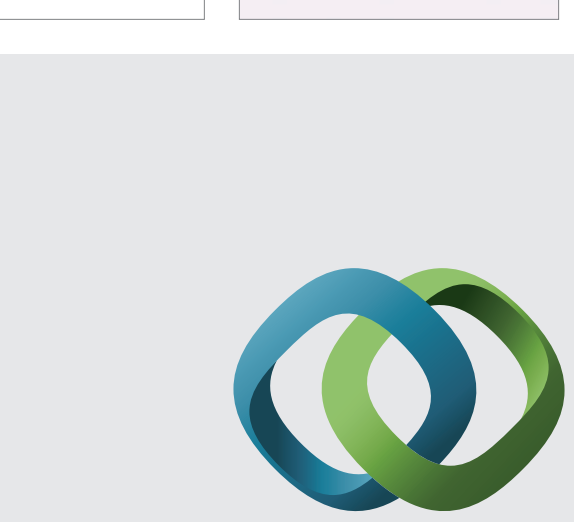

\section{Hindawi}

Submit your manuscripts at

http://www.hindawi.com
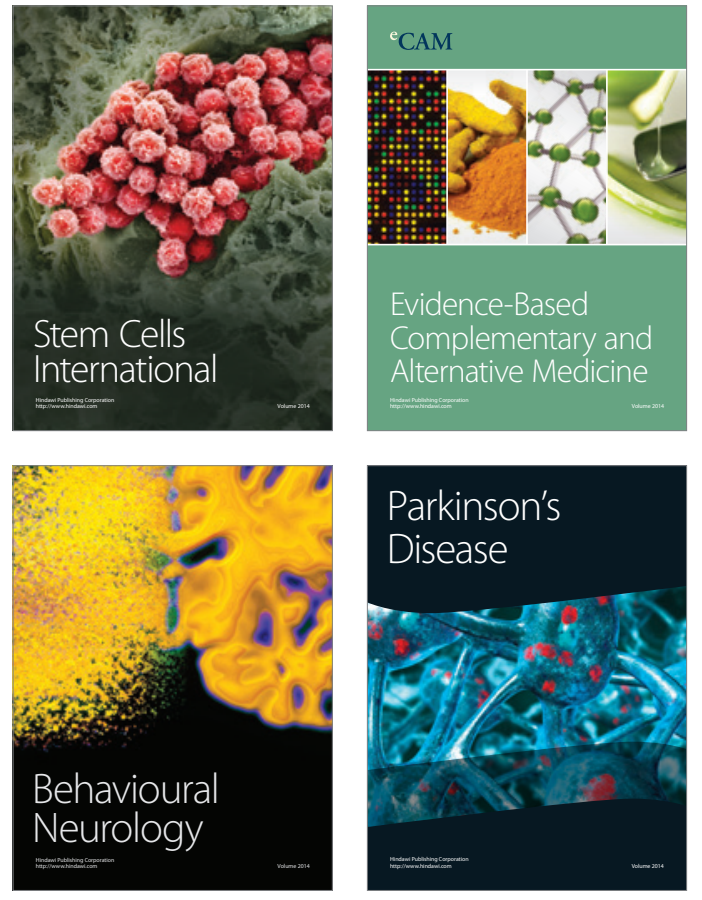
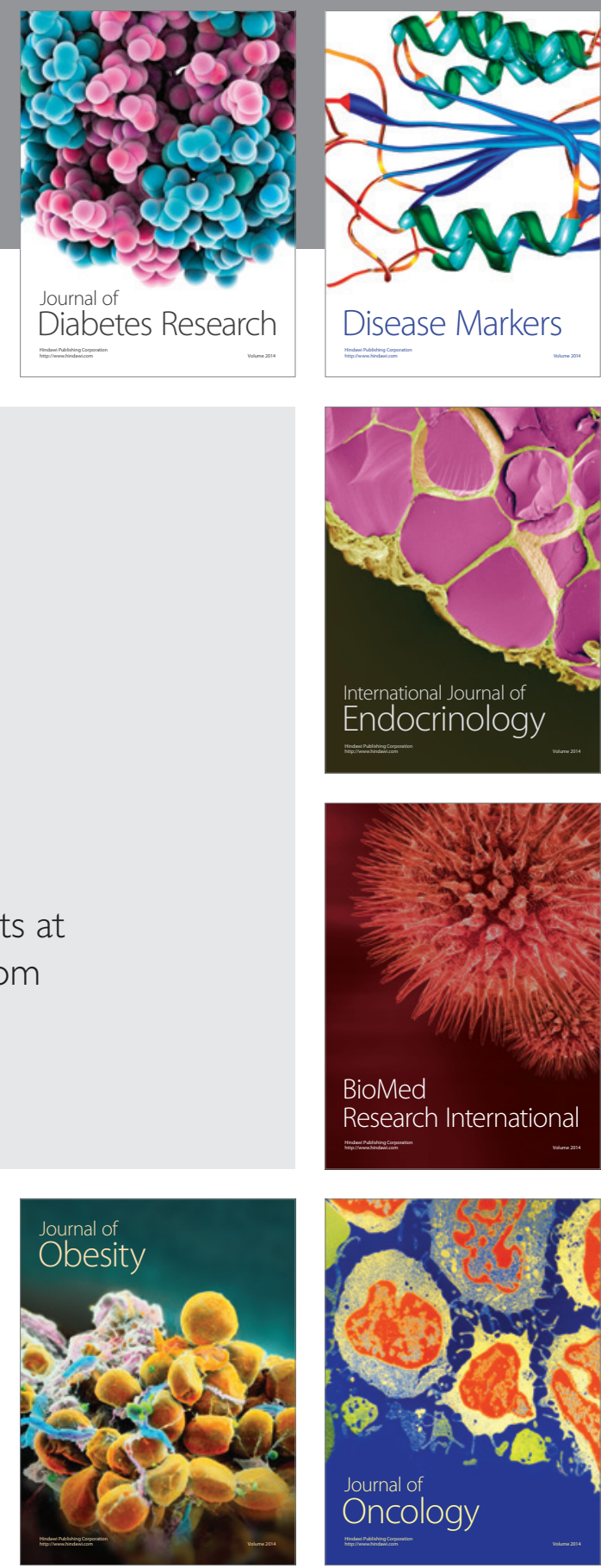

Disease Markers
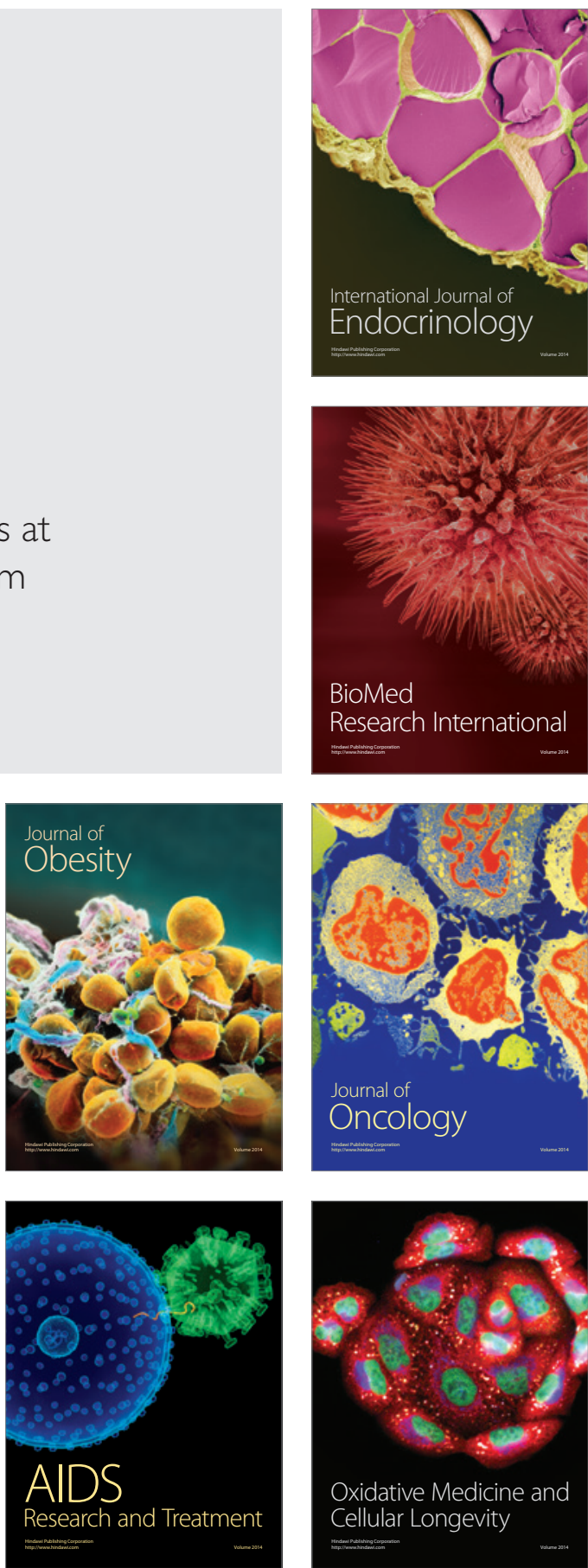